\title{
Assessment of different 3D culture systems to study tumor phenotype and chemosensitivity in pancreatic ductal adenocarcinoma
}

\author{
KATRINE ZEEBERG $^{1 *}$, ROSA ANGELA CARDONE ${ }^{1 *}$, MARIA RAFFAELLA GRECO $^{1}$, MARA SACCOMANO $^{3}$, \\ ASBJØRN NØHR-NIELSEN ${ }^{2}$, FRAUKE ALVES ${ }^{3,4}$, STINE FALSIG PEDERSEN ${ }^{2}$ and STEPHAN JOEL RESHKIN ${ }^{1}$ \\ ${ }^{1}$ Department of Biosciences, Biotechnology and Biopharmaceutics, University of Bari, Bari, Italy; ${ }^{2}$ Section for Cell \\ and Developmental Biology, Department of Biology, Faculty of Science, University of Copenhagen, Denmark; \\ ${ }^{3}$ Max-Planck-Institute of Experimental Medicine; ${ }^{4}$ Department of Haematology and Medical Oncology, \\ Institute of Diagnostic and Interventional Radiology, University Medical Center Goettingen, Goettingen, Germany
}

Received February 2, 2016; Accepted April 13, 2016

DOI: $10.3892 /$ ijo.2016.3513

\begin{abstract}
Pancreatic ductal adenocarcinoma (PDAC) is a highly malignant disease with a very poor prognosis, due to the influence of the tumor stroma, which promotes tumor growth, early invasion and chemoradiation resistance. Efforts to develop models for identifying novel anticancer therapeutic compounds have been hampered by the limited ability of in vitro models to mimic these in vivo tumor-stroma interactions. This has led to the development of various threedimensional (3D) culture platforms recapitulating the in vivo tumor-stroma crosstalk and designed to better understand basic cancer processes and screen drug action. However, a consensus for different experimental 3D platforms is still missing in PDAC. We compared four PDAC cell lines of different malignancy grown in 2D monolayers to three of the more commonly used 3D techniques (ultralow adhesion concave microwells, Matrigel inclusion and organotypic systems) and to tumors derived from their orthotopic implantation in mice. In these $3 \mathrm{D}$ platforms, we observed that cells grow with very different tumor morphologies and the organotypic setting most closely resembles the tumor cytoarchitecture obtained by orthotopically implanting the four cell lines in mice. We then analyzed the molecular and cellular responses of one of these cell lines to epidermal growth factor receptor (EGFR) stimulation with EGF and inhibition with erlotinib and found that only in the 3D platforms, and especially the organotypic, cells: i) responded to EGF by changing the expression of signalling components underlying cell-stroma crosstalk and
\end{abstract}

Correspondence to: Dr Rosa Angela Cardone, Department of Bioscience, Biotechnology and Biopharmaceutics, University of Bari, Via Amendola 165/A, I-70126 Bari, Italy

E-mail: rosaangela.cardone@uniba.it

*Contributed equally

Key words: pancreatic ductal adenocarcinoma, organotypic, spheroids, ECM, epidermal growth factor receptor, erlotinib tissue architecture, growth, invasion and drug resistance (E-cadherin, EGFR, ezrin, $\beta 1$ integrin, NHERF1 and HIF-1 $\alpha$ ) similar to those reported in vivo; ii) had stimulated growth and increased erlotinib sensitivity in response to EGF, more faithfully mimicking their known in vivo behaviour. Altogether, these results, indicate the organotypic as the most relevant physiological 3D system to study the complex tumor stroma interactions driving progression and determining chemioresistance.

\section{Introduction}

Animal models have traditionally offered an important platform for determining tumor characteristics and testing drug efficacy and dynamics in a complete physiological environment. However, the differences between mice and humans (1), together with animal models being expensive, difficult and ethically not sustainable for large drug screens have fostered the use of in vitro culture systems for pre-animal testing. While 2D monolayers have been the cornerstone of preclinical cancer research, there is increasing evidence that cells grown in 2D monolayers do not accurately reflect the biological complexity of tumors. Indeed, most if not all drugs that pass preclinical in vitro testing fail in the patients since $2 \mathrm{D}$ cultures lack, in large part, the complex stroma-cancer interactions (cell:cell and cell:matrix interactions), tissue architectures (1), and intratumoral gradients in $\mathrm{pH}$, nutrition and oxygenation found in cancers in vivo (2).

This is of particular importance for cancers, such as the pancreatic ductal adenocarcinoma (PDAC), whose tumor microenvironment has a dominant role in carcinogenesis, metastatic spread and therapeutic resistance (3). Indeed, among all cancer types PDAC, due to the strong interplay between tumor cells and stromal components, exhibits the most dense desmoplastic stroma which can account for up to $90 \%$ of the total tumor volume (4). These complex stroma-cancer interactions in PDAC result in and contribute to the inherently aggressive disease biology and a pronounced resistance to conventional therapeutic regimens. This makes PDAC one of 
the few human malignancies with a median survival time of $<6$ months, a 5-year survival rate of $5-7 \%(5,6)$ and a projection to become the second leading cause of cancer-related deaths in the next 10-15 years (7). It is, therefore, critical to establish innovative and more physiologically relevant in vitro experimental models allowing the study of drug efficacy and dynamics in the context of the desmoplastic tumor-supportive PDAC environment.

It is now generally recognized that three-dimensional (3D) cultures could be a relevant pre-clinical model with advantages over $2 \mathrm{D}$ monolayers as they more accurately reflect the architecture and bio-mechanical properties of the tumor. Indeed, cell growth dynamics and response to both growth factors and therapeutic treatment are quite different assessed in 3D models compared to 2D models (8). As a result, there has been increasing focus on developing 3D techniques and many different platforms have been proposed, all with different grades of complexity and expression of tumor environmental conditions. While the different organoid model platforms used in PDAC research have been described in a review (9), there has been no systematic comparison and validation in a single study of the differences in such systems for evaluating PDAC cell growth and response to factors increasing growth or antineoplastic agents. Therefore, it remains unclear which models best recapitulate different aspects of in vivo tumor biology and response to therapy.

Here, we describe the comparative analyses of $2 \mathrm{D}$ culture and a series of 3D PDAC cancer microtissue models obtained culturing PDAC cells in ultra-low adhesion, concave microwell plates, implanting them in reconstituted Matrigel drops, or on Matrigel in organotypic culture to model disease pathogenesis and drug pharmacodynamics. We used as a read-out their molecular and cellular responses when the PDAC-driver epidermal growth factor receptor (EGFR) is stimulated and/or inhibited, respectively with EGF and the small tyrosine kinase inhibitor (TKI) of EGFR, erlotinib. We conclude that the organotypic system most closely mimicked the complex biological interactions driving progression and determining drug sensitivity.

\section{Materials and methods}

Cell lines and culture platforms. Experiments were performed on the following well established human pancreatic cancer cell lines as reported (10): Panc-1, BxPC3, MiaPaCa-2 and CAPAN-2. All cells were kept at $37^{\circ} \mathrm{C}$ in humidified air containing $5 \% \mathrm{CO}_{2}$. PANC-1 cells were grown in bicarbonatebuffered Dulbecco's minimal essential medium (DMEM; $\mathrm{pH} 7.4$, stable glutamine, $4.5 \mathrm{~g} / 1$ glucose). MiaPaCa-2 was grown in bicarbonate-buffered Dulbecco's minimal essential medium/Ham's F12 (DMEM/F-12; pH 7.4, stable glutamine, $4.5 \mathrm{~g} / 1$ glucose). BxPC3 and CAPAN-2 cells were cultured in bicarbonate-buffered RPMI-1640 medium [Gibco 52400-025, pH 7.4, stable glutamine $(2 \mathrm{mM})]$. Media were always supplemented with $10 \%$ heat-inactivated fetal calf serum (FCS, PAA Gold) and $1 \%$ penicillin and streptomycin. MiaPaCa-2 cells were further supplemented with $2.5 \%$ heat-inactivated horse serum (Biochrom, Germany).

Antibodies and reagents. Primary antibodies were purchased from: EGFR (Cell Signaling Technology, rabbit cat no.
4267), p(1173)EGFR (GeneTex, rabbit monoclonal cat no. GTX1052), HIF1 $\alpha$ (BD, rabbit cat no. 610959), $\beta 1$ integrin (Santa Cruz, mouse cat no. sc-18887) ezrin (BD, mouse cat no. 610603) p(T567)ezrin (Abcam, rabbit cat no. ab47293), NHERF1 (BD mouse cat no. 611160), E-cadherin (R\&D Systems, mouse cat no. 180215) and $\beta$-actin (Sigma, mouse cat no. A5441), respectively. Secondary antibodies were anti-mouse (Sigma) and anti-rabbit (Cell Signaling). Humanrecombinant epidermal growth factor (EGF) (PreproTech, NJ, USA) was dissolved in $\mathrm{H}_{2} \mathrm{O}$ and used at a final concentration of $100 \mathrm{ng} / \mathrm{ml}$. The EGFR inhibitor erlotinib (Selleckchem, Italy) was solubilized in DMSO.

Spheroid-forming assay. Cells were seeded (2,500 cells/well) in 96-well ultra-low adhesion round bottom plates (Corning Costar Sigma-Aldrich, Italy) that will be referred to here as concave microwells. The well's shape forces all the cells to collect at the bottom of the well where they adhere to each other, forming loose or compact spheroids depending on cell type. Here the spheroids are therefore non-clonal in that each was comprised of all the cells seeded. The wells of the outer edge of the plate were filled with culture medium to prevent an uneven evaporation. Pharmacological treatments were added the next day and maintained for a total of 7 days with a midweek change of medium, including treatments if present.

Colony formation in 3D Matrigel inclusion. To analyse the ability of cells to grow in an anchorage-independent manner in 3D semi-solid media (ECM scaffolds) (11), we dispersed 10,000 cells of each cell line into $10 \mu 1$ drops of Matrigel (BD Bioscience) mixed with serum-free culture medium to a final concentration of $7 \mathrm{mg} / \mathrm{ml}$. The drop of Matrigel was placed in the middle of the well of a 24 -well plate which was then inverted for the first $30 \mathrm{~min}$ of polymerization, entrapping the cells in the drop and preventing collection of cells at the bottom. The plate was then turned right side-up and allowed to polymerize for an additional $30 \mathrm{~min}$ in a $37^{\circ} \mathrm{C}$ incubator and $400 \mu \mathrm{l}$ culture medium was added and changed every 3 days. As each cell line had different growth rates the colonies were grown for 12, 8, 6 and 4 days for CAPAN-2, BxPC3, PANC-1 and $\mathrm{MiaPaCa}-2$, respectively. Here the many spheroids that formed are clonal as each grew from a single dispersed cell that then grew into a colony.

Organotypic $3 D$ culture. Cells were seeded at a density of 15,000 cells/well of 96-well plates on top of an extracellular matrix gel prepared by mixing Matrigel (BD Bioscience) with serum-free culture medium, to a final concentration of $7 \mathrm{mg} / \mathrm{ml}$. One hundred microliters/well was plated into 96-well plates and incubated for $60 \mathrm{~min}$ in a $37^{\circ} \mathrm{C}$ incubator allowing the gels to solidify. In-order-to ensure proper attachment of the cells, treatments where added the next day and growth was maintained for a total of 7 days, with a midweek change of medium.

Assessment of growth reduction by resazurin and integrated density. Cell viability was measured using the resazurin cell viability assay (Immunological Sciences). The resazurin method is an easy and fast assay to measure cell viability and is based on the principles of the MTT assay, but with the superiority of not 
having to extract the dye from the cells. Resazurin (10 $\mu \mathrm{l})$ was added to each $100 \mu \mathrm{l}$ of medium according to the manufacturer's instructions and fluorescence was measured after $\sim 3 \mathrm{~h}$. Relative cell number was calculated from standard curves of resazurin fluorescence vs cell number in a Burker chamber and growth or response to erlotinib measured as the change in cell number over time.

In the experiments with ultra-low adhesion concave plates and Matrigel inclusion drops, images of the spheroids from both culture systems were acquired and analyzed with a $60 X$ oil objective using a Nikon Eclipse TE 2000S epifluorescence microscope. The spheroids were analyzed for size, circularity and integrated density using ImageJ (http://rsb.info. nih.gov/ij/). For integrated density, images of the cultures were uploaded to the program, colors were inverted and the images were converted into 8-bit. Threshold was adjusted to have only the cell mass emerge and integrated density value was then calculated by multiplying mean grey values by area.

Western blot analysis. Cells were lysed directly from 2D monolayers and ultra-low adhesion concave microplates while in Matrigel inclusion drops and the organotypic culture cells were extracted from the Matrigel matrix by the use of CellSperse (Cultrex) and lysed in lysis buffer (HEPES $5 \mathrm{mM}$, EDTA $0.5 \mathrm{mM}, \mathrm{pH} 7.2$ supplied with protease inhibitor $2 \mu \mathrm{l} / \mathrm{ml}$, phenylmethanesulfonylfluoride (PMSF) $1 \mathrm{mM}$, sodium orthovanadate $1 \mathrm{mM}$, dithiothreitol (DTT) $1 \mathrm{mM}$, Nonidet 0.1\%). Proteins were measured with Bradford (Pierce), resuspended in sodium dodecyl sulfate (SDS) sample buffer [6.25 mM Tris- $\mathrm{HCl}$, $\mathrm{pH} 6.8$, containing $10 \%$ (v/v) glycerol, $3 \mathrm{mM}$ SDS, $1 \%(\mathrm{v} / \mathrm{v})$ 2-mercaptoethanol and $0.75 \mathrm{mM}$ of bromophenol blue], run on $10 \%$ SDS-PAGE and blotted to Immobilon P. The protein expression levels of EGFR, p(1173)EGFR, HIF-1 $\alpha$, Ezrin/p(T567)Ezrin, $\beta 1$ integrin, NHERF1 and E-cadherin were analyzed with their primary antibodies. Each blot was scanned with an Epson V600 scanner and the relative optical density of each band was analyzed using ImageJ (http://rsb.info. nih.gov/ij/).

Orthotopic implantation of human pancreatic tumor cell lines and $H \& E$ staining of tissue specimens. Cells of the PDAC cell lines were implanted orthotopically in severe combined immunodeficient mice (SCID mice), strain C.B-17/Ztm-scid of both sexes or nude mice, strain NMRI-Fox1 nu/nu, as described (10). Animal studies adhere to the Animal Welfare Act in the version published on 18 May 2006 (Federal Law Gazette I p. 1206, 1313) amended by article 4 section 90 of the Act of 7 August 2013 (Federal Law Gazette I p. 3154) which is in full correspondance with European legislation. Tumor cell implantation was performed in accordance with the Declaration of Helsinki protocols as described previously (12). Professor F. Alves and her team, supported by the veterinarian S. Kimmina, have approval for ongoing research in oncology including orthotopic pancreatic tumor models using experimental mice (Tierversuchsantrag: 33.42502/103/06 and 33.9-42502-04-13/1085) from the Niedersachsen animal welfare committee (the respective local institution: Niedersächsisches Landesamt für Verbraucherschutz und Lebensmittelsicherheit). For orthotopic transplantation, general anesthesia was performed by intraperitoneal application using a ketamine-xylazin mixture (75-100/15-20 mg/kg b.w.). A median laparotomy was performed, the peritoneum opened and the pancreas carefully exposed. Aliquots of $1 \times 10^{6}$ pancreatic tumor cells in a volume of $15 \mu \mathrm{l}$ PBS were injected very slowly with an insulin syringe into the duodenal lobe of the pancreas through the pancreatic serosa into the pancreatic tissue. The pancreas was then returned to the abdominal cavity and the incision closed in two layers using Vicryl suture (Metric 1.5, Ethicon, Norderstedt, Germany). After implantation, mice were monitored at least five times a week following tumor cell transplantation by expert personnel through direct observation of fur signs, tumor development, abdominal distension and weight loss. Weight loss exceeding $20 \%$ of the initial weight that lasts more than two days was the criteria for experimental termination. For post-surgical analgesia treatment, mice received analgesic (i.p.; Rimadyl; active substance: carprofen; doses $5 \mathrm{mg} / \mathrm{kg}$ b.w.). Operated animals were kept on a warming device. Animals were anesthesized by isoflurane, and finally painlessly euthanized by cervical dislocation. The pancreatic tumor masses were excised and placed in phosphate-buffered $4 \%$ formalin for $16 \mathrm{~h}$ at room temperature and embedded in paraffin. Tissue sections $(2.5 \mu \mathrm{m})$ were obtained and stained with hematoxylin and eosin (H\&E) using standard protocols.

Statistical procedures. Data correspond to at least three independent experiments, each of which was done in triplicate. Results are presented as means \pm standard error of the mean (SEM). The results of erlotinib +/- EGF treatment on $3 \mathrm{D}$ growth were analyzed according to published methods (13). The data for each condition were subject to analysis of variance (ANOVA) followed by Dunnet post hoc test when comparing three or more conditions or evaluated using Student's t-test when comparing only two conditions. Differences were considered significant with values of $\mathrm{P}<0.05$. The results of treatments with erlotinib on 3D growth were analyzed in KaleidaGraph-Synergy software (Reading, PA, USA) using the median effect equation, $\mathrm{fa} / \mathrm{fu}=(\mathrm{D} / \mathrm{Dm})^{\mathrm{m}}$, in which fa is the fraction affected by dose $D, f_{u}$ is the fraction unaffected, $D$ is the dose and $\mathrm{D}_{\mathrm{m}}$ is the dose required for $50 \%$ growth inhibition and $m$ is the coefficient of sigmoidicity (13).

\section{Results}

Characterization and comparison of tumor morphology and growth in $2 D$ versus $3 D$ systems and in vivo. Cells grown on 2D tissue culture substrates differ considerably in their morphology and differentiation and their cell-cell and cellmatrix interactions from cells in vivo $(2,14,15)$. While it is known that both intrinsic 3D architecture and the ECM exert strong effects on cell morphology, a single study comparing the effects of different 3D systems on PDAC cell morphology, growth, molecular signaling and response to therapy has yet to be done. Here, we first analyzed the impact of 2D and three known 3D tissue cultures, namely the ultralow adhesion concave microwells, Matrigel inclusion and the organotypic system, in affecting basal growth and morphology of a panel of PDAC cell lines (Fig. 1) and the resulting growth patterns were compared with those obtained by orthotopically implanting the same cell lines in mice. We utilized BxPC3 cells, which 

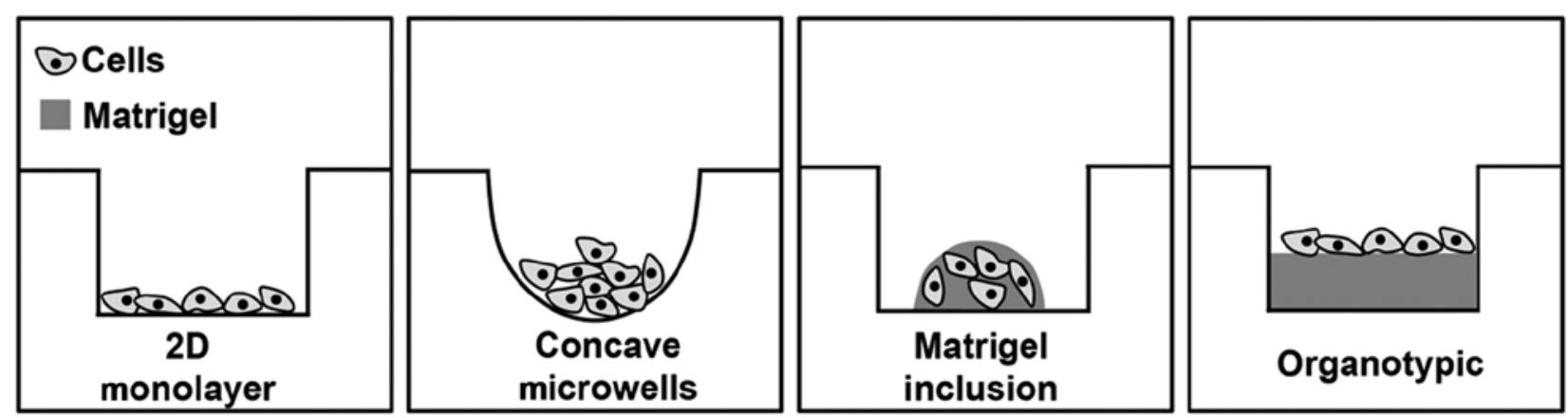

Figure 1. Schematic overview of pancreatic tumor cell culture platforms. Pancreatic ductal adenocarcinoma (PDAC) cells were cultured in a variety of platforms ranging from the classical 2D monolayer on cell culture plastic, in ultra-low adhesive concave microwells, dispersed as single cells in 7 mg/ml Matrigel (Matrigel inclusion) or seeded on $7 \mathrm{mg} / \mathrm{ml}$ Matrigel in an organotypic system.

carry p53 mutations, and PANC-1 and MiaPaCa-2, which are poorly differentiated and carry both KRAS and p53 mutations (16). In addition, CAPAN-2 cells were included as it is a welldifferentiated PDAC cell line (17). The BxPC3 and CAPAN-2 lines have been classified as less aggressive, classical PDAC cell lines while PANC-1 and MiaPaCa-2 are very aggressive lines of the quasi-mesenchymal QM-PDAC type (16).

The morphological growth patterns of the four cell lines in all four culture systems from less (left) to more (right) aggressive are shown in Fig. 2A. When grown in 2D, all four cell lines grew as monolayers but displayed different morphologies, such that CAPAN-2 and BxPC3 grew as tight cobblestone monolayers, while both PANC-1 cells and the very aggressive MiaPaCa- 2 cells formed monolayers composed of loose aggregates with a high percentage of cells having an elongated, mesenchymal form. Cells grown in 3D spheroidproducing techniques generated a single multiclonal spheroid with the ultra-low adhesion concave microwells system and a high number of monoclonal spheroids with the Matrigel inclusion method, but in both systems colonies formed with similar shapes and dynamics independently of the presence of an extracellular matrix. However, spheroid-growth patterns, measured by integrated density analyses and circularity indexes were quite different among each cell line and consistent with their reported malignant potential and subtype gene signatures (16). The spheroids from the less aggressive, classical PDAC cell lines (CAPAN-2 and BxPC3) increased their size slower (Fig. 2A) and had a more spherical shape (Fig. 2B; circularity index of $0.93 \pm 0.04$ and $0.96 \pm 0.02$, respectively). In contrast, spheroids from the more aggressive, PANC-1 and MiaPaCa-2 cells grew larger in less time (Fig. 2A) and had a more irregular morphology (Fig. 2B, basal circularity index of $0.87 \pm 0.06$ and $0.83 \pm 0.05$, respectively). Interestingly, the circularity index of each cell line was not significantly different between spheroids developed in the concave microwell and Matrigel inclusion systems (Fig. 2B).

Only when grown in organotypic culture (Fig. 2A, fourth row), where the cancer cells are seeded on top of the matrix, was there a strong phenotypic variability in microtissue tumor morphology between the cell lines, such that the least aggressive cell line, CAPAN-2 (16), formed a monolayer rich in globular structures, the BxPC3 formed a vascular type network while the more aggressive PANC-1 and MiaPaCa-2 lines initially formed irregular colonies that eventually coalesced into complex microtissues. As this increased phenotypic variability in morphology probably more closely reflects the in vivo characteristics of the different lines, we compared these observed morphological differences with PDAC in vivo, by implanting the four PDAC cell lines orthotopically into the pancreas of nude mice and analyzing the morphological patterns of pancreatic tumor sections obtained by hematoxylin and eosin staining (H\&E) [Fig. 2A, fifth row; (10)]. Keeping in mind the much higher complexity in vivo, the general morphology of the tumors in all the tumor sections closely followed that observed in the organotypic culture system. The CAPAN-2 and BxPC3 cell lines grew in vivo to become, respectively, a well and moderately differentiated tumor, as confirmed by the presence of in vivo duct-like structures (asterisks) with a distinct apical-basal polarization. Moreover, in vivo growth of $\mathrm{BxPC} 3$ cells formed organized islands of tumor cells (hashtag character) and the presence of small vessels lined with tumor cells and containing erythrocytes (arrows), suggesting that, indeed, this cell line is able to form microvascular-like bed complexes in vivo. On the contrary, tumors derived from PANC-1 and MiaPaCa-2 cell lines developed undifferentiated primary tumor masses, which proliferated in an unorganized way in vivo.

Expression of tissue architecture, growth signaling and microenvironment proteins. As cells grow in $3 \mathrm{D}$, they alter the expression of a number of proteins regulating tissue architecture, matrix interaction and growth factor signaling $(14,15,18)$. We therefore determined the effect of the different 3D culture systems on basal and EGF stimulated $(100 \mathrm{ng} / \mathrm{ml}$, $24 \mathrm{~h}$ ) expression in western blotting of key proteins regulating cell-cell and cell-matrix interactions, epithelial to mesenchymal transition (EMT) and metastasis (Fig. 3A). For this, we utilized the PANC-1 cell line since it is known to be the most resistant to drugs targeting the EGFR (19). We first looked at E-cadherin together with $\beta 1$ integrin, EGFR and ezrin, a membrane-cytoskeleton linker protein, in virtue of their known involvement in mediating cell-ECM interactions, EMT and invasion in PDAC (20). In basal conditions, the expression of E-cadherin (Fig. 3B), a marker of epithelial tissue organization, was higher in all the 3D systems compared to 2D culture, while $\beta 1$ integrin (Fig. 3D) expression was high only in the 

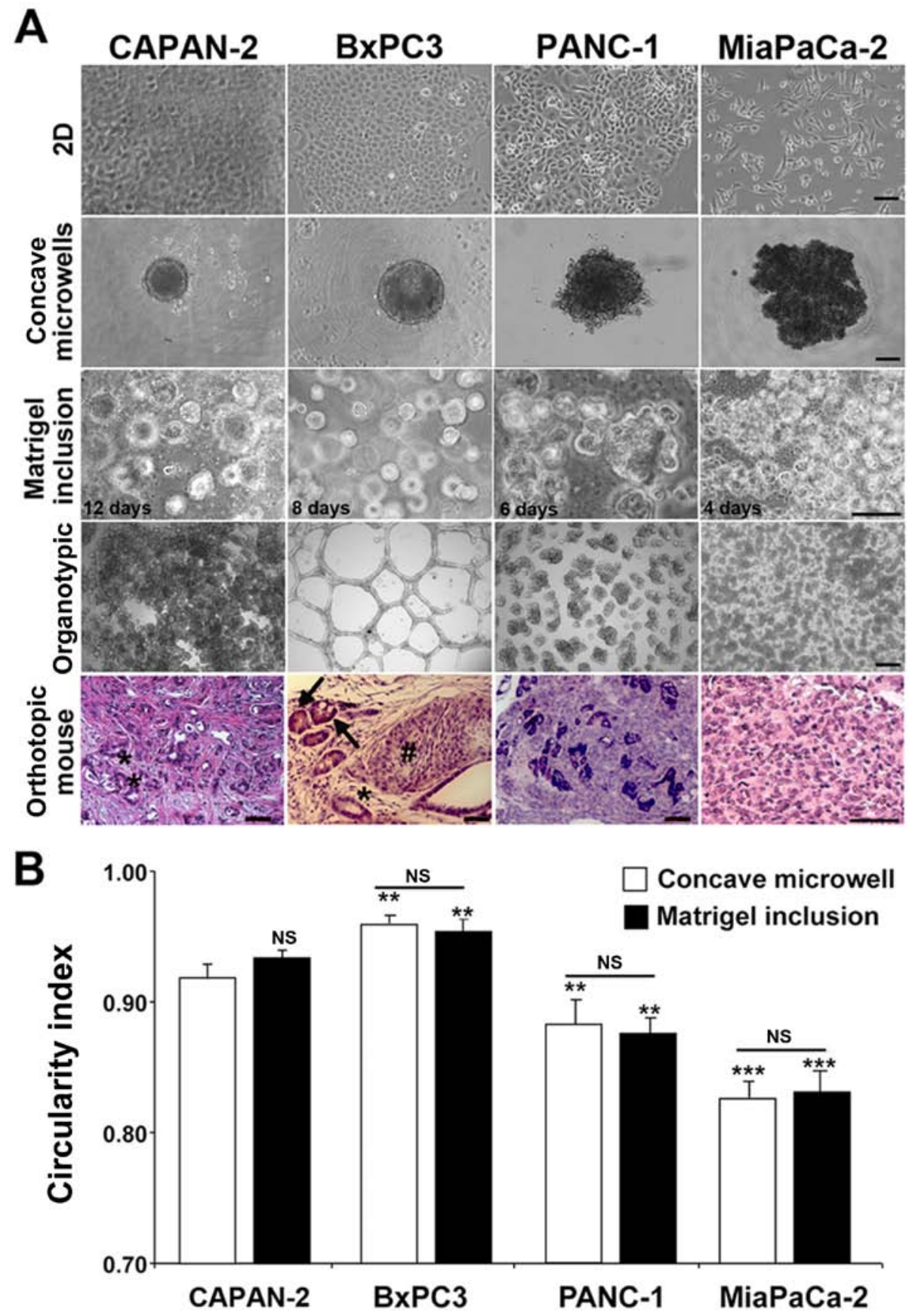

Figure 2. Morphological comparisons of PDAC cells in 2D and different 3D systems. PDAC cells were cultured in the different platforms and images of representative structures were captured at different time-points as indicated in the images. (A) Representative images of colonies from the four PDAC cell lines in 2D (first row), ultra-low adhesion concave microwells without ECM (second row), dispersed in $7 \mathrm{mg} / \mathrm{ml}$ Matrigel (third row), in organotypic (forth row) and hematoxylin and eosin (H\&E) staining in pancreatic tissue sections derived from immunosuppressed mice in which the four PDAC cell lines were orthotopically implanted (fifth row). Only cells in the organotypic 3D culture conditions showed a close resemblance to the in vivo orthotopic tumors developed from the same cell lines. Scale bars, $10 \mu \mathrm{m}$ for 2D cultures; $25 \mu \mathrm{m}$ for all three 3D cultures and $50 \mu \mathrm{m}$ for in vivo tumors. Asterisks indicate duct-like structures with a distinct apical-basal polarization, the hashtag character indicates a dense mass of tumor cells and arrows indicate small vessels lined with tumor cells and containing erythrocytes. Images in Matrigel inclusion (second row) were taken at different days after plating (12, 8, 6 and 4 days for CAPAN-2, BxPC3, PANC-1 and MiaPaCa-2, respectively) because of the large differences in colony growth. (B) Colony circularity index measured and calculated in Fiji. Data are shown as mean \pm SEM for four independent experiments; ANOVA followed by Dunnet post hoc test: ${ }^{* *} \mathrm{P}<0.01 ;{ }^{* * *} \mathrm{P}<0.001$ of the circularity index of the spheroids of the different cell lines compared to the CAPAN-2 cell line spheroids in each respective culture system. The circularity index for each cell line was not significant (NS) between Matrigel inclusion (black bars) and their respective spheroids in concave microwells (open bars).

presence of matrix and was higher in organotypic than in Matrigel inclusion. Importantly, only in 3D culture conditions, and especially in the organotypic platform, did EGF treatment increase both EGFR phosphorylation (Fig. 3E) and $\beta 1$ integrin
(Fig. 3D) expression while decreasing E-cadherin expression (Fig. 3B). This supports previous reports that E-cadherin expression decreases during EMT, invasion and metastasis (21-23) and that reduced E-cadherin expression is associated 
A

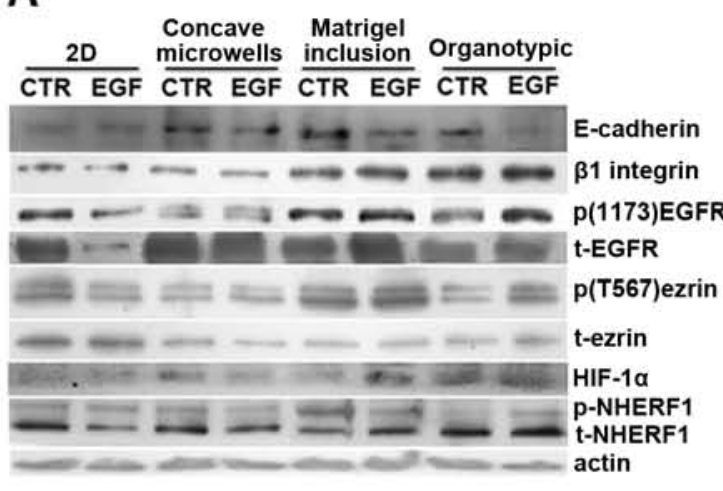

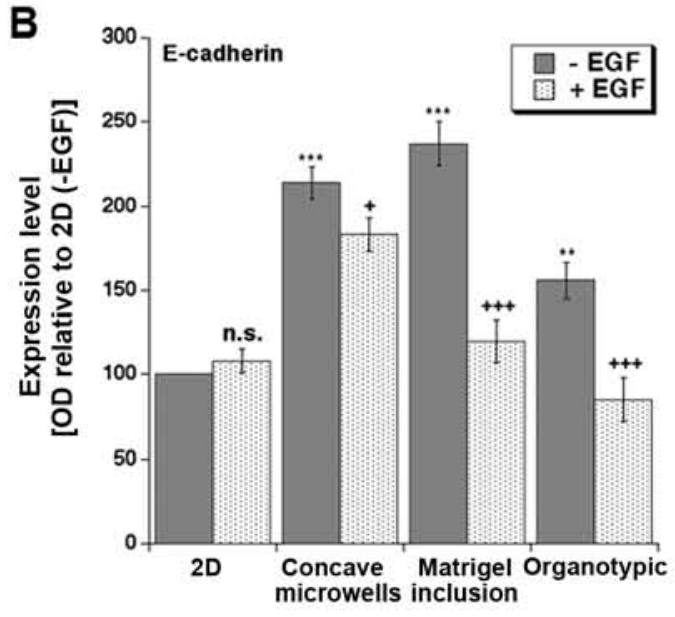

D

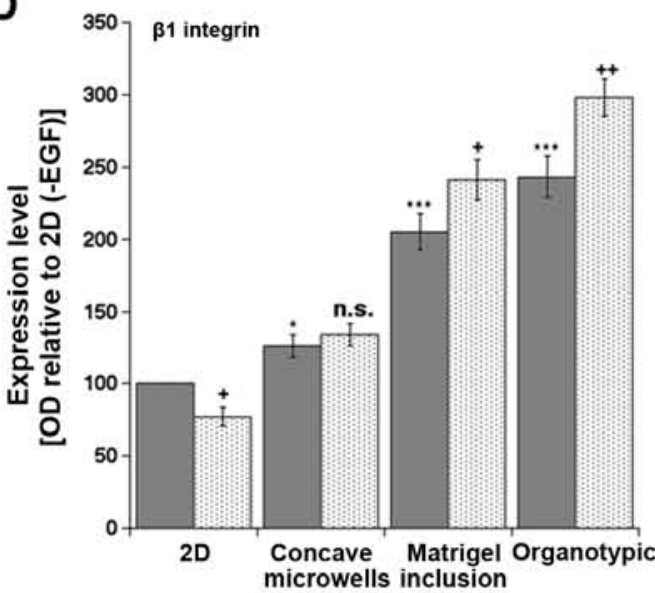

$\mathbf{F}$

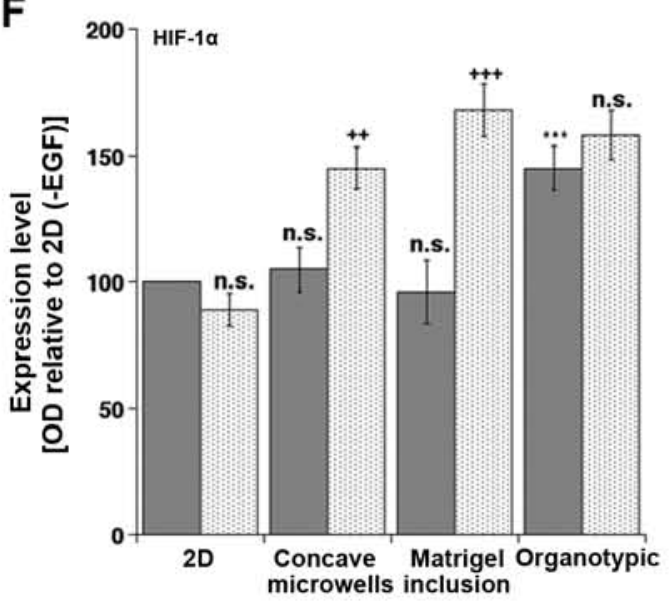

Figure 3. Effects of culture system and EGF on protein expression levels of p-EGFR, EGFR, HIF-1 $\alpha$, ezrin, p-ezrin, $\beta 1$ integrin, NHERF1 and E-cadherin. (A) Typical blots of PANC-1 cells cultured for three days in the different platforms in the absence or presence of EGF $(100 \mathrm{ng} / \mathrm{ml})$. The collected homogenates were separated in SDS-PAGE, transferred to Immobilon $P$ transfer membranes and the expression of E-cadherin, $p(T 567)$ ezrin, total ezrin, $\beta 1$ integrin, $\mathrm{p}$ (1173)EGFR, EGFR, HIF-1 $\alpha$, and NHERF1 were analyzed by western blotting with their primary antibodies as described in Materials and methods. The expression levels were analyzed in ImageJ as described in Materials and methods and standardized for actin levels. (B-G) Histograms summarizing the relative expression levels from 4 independent experiments using the expression levels of the 2D minus EGF treatment (2D, -EGF) as $100 \%$ and expressed as mean \pm SEM for E-cadherin, $p$ (T567)ezrin, $\beta 1$ integrin, pEGFR, HIF- $1 \alpha$ and pNHERF1, respectively. Statistical analysis was ANOVA followed by Dunnet post hoc test: ${ }^{* *} \mathrm{P}<0.01 ;{ }^{* * * *} \mathrm{P}<0.001$ of optical density of the (-EGF) band for each protein in each 3D system compared to that in $2 \mathrm{D}$ culture while ${ }^{++} \mathrm{P}<0.01$; ${ }^{++} \mathrm{P}<0.001$ between the $-\mathrm{EGF}$ and + EGF treated cells for each system. NS, not significant. 

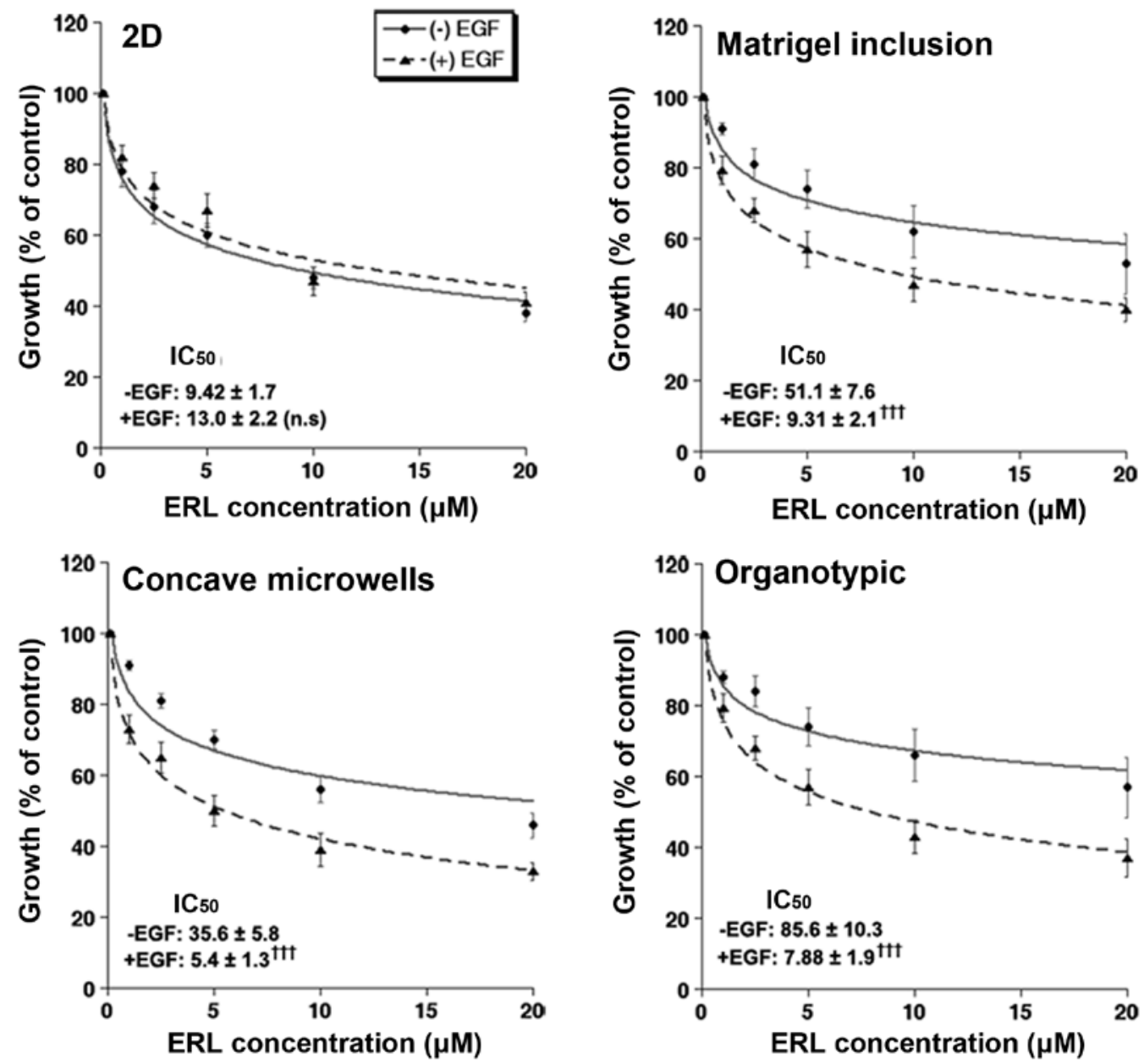

Figure 4. Dose-response curves of erlotinib and EGF on PDAC cell growth in the four culture systems. All experiments were conducted in PANC-1 cells in the absence or presence of $100 \mathrm{ng} / \mathrm{ml}$ EGF and in increasing erlotinib concentrations for five days. Growth was measured using the resazurin cell viability assay as described in Materials and methods. While EGF treatment increased the growth rate in all 3D platforms, growth rates in the absence of erlotinib of both plus and minus EGF are shown as $100 \%$ in-order-to facilitate visual comparison of the dose responses of each treatment. Data are shown as mean \pm SEM of three or four independent experiments performed in triplicate. Curve fitting was performed for each curve and $\mathrm{IC}_{50}$ values in $\mu \mathrm{M}$ are the mean $\pm \mathrm{SEM}$ calculated as per Chou (13); ${ }^{\dagger \dagger} \mathrm{P}<0.001$ of cells without EGF comparedto cells incubated with EGF.

with increased $\beta 1$ integrin levels in breast (24) and advanced ovarian (25) cancers. In line with the phosphorylation of ezrin on threonine 567 (p(T567)ezrin) being increased in several human tumor tissues and playing a role in metastasis by favoring motility and invasion (21), we found that $\mathrm{p}$ (T567)ezrin, normalized to total ezrin expression, was downregulated by EGF in 2D culture, not regulated in concave microwells and Matrigel inclusion and strongly upregulated only in the organotypic culture (Fig. 3C). Thus, only the organotypic platform confirmed the in vivo reports of a correlation of increased $\mathrm{p}$ (T567)ezrin levels, which requires activated $\beta 1$ integrin receptor, with the downregulation of E-cadherin (20).

We then extended the analysis to some signaling proteins important in transducing the effects of the tumor microenvironment. First of all, we found that the hypoxia marker, HIF-1 $\alpha$, which in human PDAC samples correlates with tumor size, aggressiveness and poor prognosis (26), exhibited the lowest protein levels in the 2D system and the highest expression in both Matrigel cultures exposed to EGF and in the organotypic cultures even in the absence of EGF. This expression pattern, which reflects the desmoplastic, hypovascularised and highly hypoxic nature of PDAC only in the organotypic model, was shared by another tumor hypoxia microenvironment-associated protein (27-30), the scaffolding protein NHERF1. Importantly, NHERF1 expression is also increased in several human cancers including PDAC (10) and it can be phosphorylated by the protein kinase A (PKA), via the PKA-anchoring activity of ezrin (31). In line with this, we detected an increase of the higher molecular weight band of NHERF1, corresponding to phospho-NHERF1, in the organotypic cultures exposed to EGF, where the increase of $\mathrm{p}$ (T567)ezrin, i.e., active ezrin, was highly significant (Fig. 3G). This finding also recapitulates the natural increase in both $\mathrm{p}$ (T567)ezrin expression and NHERF1 phosphorylation that have been already reported in human tumor tissues $(21,32)$ and further validates the organotypic system as the more relevant 3D approach to study PDAC biology in response to its microenvironment.

$3 D$ culture promotes sensitivity to EGF and resistance to erlotinib differently for the various culture systems. One of the many characteristics of cancer cells grown in 3D culture is increased chemo- and radio-resistance to anticancer therapy compared to that in $2 \mathrm{D}$ culture $(33,34)$. It is considered that 
both 3D architecture and ECM exert strong effects on both the regulation of growth and drug efficiency (35). To measure the effect of EGF on cell sensitivity to the EGFR tyrosine kinase inhibitor, erlotinib, we treated PANC-1 cells with various concentrations of erlotinib in the presence or absence of EGF and measured cell growth using the resazurin assay and transforming to cell number. Only the cells cultured in $3 \mathrm{D}$ responded to EGF addition with an increase in cell number of $32 \pm 7.9 \%(n=8), 29 \pm 12.4 \%(n=7)$, and $26 \pm 8 \%(n=5)$ in the concave microwell, Matrigel inclusion and organotypic systems, respectively. Similarly, as can be seen in Fig. 4, cells grown in all three 3D systems were more resistant to erlotinib than in the 2D system with the cells grown in the organotypic system being the most resistant in basal conditions. While EGF treatment increased the growth rate in all 3D platforms, control growth rates (in the absence of erlotinib) are shown as $100 \%$ in-order-to facilitate visual comparison of the dose responses of each treatment. Interestingly, these differences in drug sensitivity are in line with the expression data as E-cadherin expression has been linked to drug resistance and clinical trials of the erlotinib in non-small cell lung cancer have shown better responses in patients with high E-cadherin expression (36). Furthermore, concomitant incubation of EGF with erlotinib significantly enhanced the inhibitory effect of erlotinib only in the 3D cultures with a greater effect occurring in the organotypic platform (Fig. 4). These inhibition data are in line with the reported increased resistance to pharmacological therapy in other 3D cell culture formats (5-7) and suggest that efficacy assays performed in physiologically relevant culture conditions become more predictive to in vivo responses.

Altogether, these data support earlier reports that characteristics of 3D cultures are similar to those in the original tumor (37) and suggest that the organotypic system most closely recapitulates the in vivo tumor characteristics in morphology, the relationships in protein expression and pharmacodynamics.

\section{Discussion}

As only $5 \%$ of compounds that show pre-clinical efficacy go on to become licensed drugs (1), more predictive in vitro efficacy and toxicity assays are needed to identify new anticancer drugs and reduce the number of costly drug failures in clinical trials. Traditionally, two-dimensional (2D) cell culture models have been employed to evaluate drug candidates in the early phases of the drug discovery process. However, cells grown in 2D monolayers do not accurately reflect the biological complexity of tumors as such cultures are a highly reductionist model of epithelial cancers and poorly represent in vivo tumor cell biology, due to the absence of relevant properties, such as cell-cell communication, extracellular matrix (ECM) contacts, differentiation, polarization and intratumoral gradients in $\mathrm{pH}$, nutrition and oxygen (i.e., a lack of realistic mass transfer gradients). For these reason, animal studies have always been utilized as the final pre-clinical passage before human experimentation, to predict drug efficacy and to understand the biological processes driving the tumor development.

However, given the differences between mice and humans $(38,39)$, techniques that can mimic in vitro the development of a human tumor have an enormous potential to further increase our understanding of the dynamics of progression, metastasis, drug response and therapy resistance. This need is further accentuated by the creation, in 2005, of the European Partnership for Alternative Approaches to Animal Testing (EPAA) and the directive of 2010/63/EU on the protection of animals used for scientific purposes that recommend to 'reduce, refine and finally replace' the use of animal experimental models. This has created an urgent need to develop new alternative tests to animals.

This requirement for better in vitro models has led to the development of a large variety of 3D cell culture systems, which retain different aspects of the morphological and physiological traits of tumors and better predict tumor behavior by mirroring complex tissue organization and the myriad of microenvironmental signals impacting tumor growth. Although there has been much progress in constructing these in vitro physio-pathological models, there is still no consensus on which 3D models of PDAC are best able to mimic the tumor's molecular (genome and proteome) and functional (chemotherapy sensitivity and signaling) characteristics. Indeed, a rigorous comparison of these different systems in a single cancer type has not been performed. Therefore, we have characterized growth, proteins involved in cell-ECM communication, tissue architecture, EMT and metastasis, and the response to the EGFR small molecule inhibitor, erlotinib, in a panel of different 3D culture systems commonly in use and having different ECM properties.

While our data collectively revealed similarities between the different 3D systems, the results presented here suggest that the organotypic 3D system has many advantages over the other systems. i) Importantly, only the organotypic culture permitted the cells to express their inherent/intrinsic different microtissue morphologies that reflected the tumor structure as observed in orthotopic tumors from each cell line (Fig. 2, fifth row). ii) At the protein level, we found some important differences among the three different culture systems with the organotypic model being the more tissue-mimetic platform also for protein expression/mechanistic studies of tumor microenvironment-induced cell signaling components. Indeed, only the organotypic system mirrored the very tight relationships already reported in vivo among E-cadherin, $\beta 1$ integrin, NHERF1, pEGFR and $\mathrm{p}(\mathrm{T} 567)$ ezrin, proteins mediating tissue architecture, cell-ECM interactions, EMT and metastasis (21-24). Furthermore, HIF-1 $\alpha$, which plays major roles in regulating the tumor response to its hypoxic microenvironment (21-24) was expressed at its very low/ basal level in the matrix-free systems, such as the 2D system and the concave microwells, while increasing only when the hypoxia-biomimetic conditions reached into the tumor spheroids, i.e., in the case of the Matrigel drops exposed to EGF and in the organotypic system. In the organotypic culture system, hypoxia-induced HIF-1 $\alpha$ expression may have already reached its maximum stabilization level, thus reducing its sensitivity to EGF stimulation. iii) We also tested the different culture systems chemosensitivity to erlotinib, and found that cells were more resistant to this drug when grown in all the $3 \mathrm{D}$ systems compared to a $2 \mathrm{D}$ monolayer but particularly in the organotypic scaffold.

Furthermore, we observed that the organotypic cultures displayed the highest resistance to erlotinib in basal condi- 
tions, but with an increased response in the presence of EGF stimulation compared to spheroids in either non-clonal concave microwell or the clonal Matrigel inclusion systems. iv) Importantly, the organotypic cultures have the capacity to produce $3 \mathrm{D}$ platforms that more faithfully reproduce and permit the experimental manipulation of the various aspects of the complete tumor microenvironment including ECM components and different cell populations (e.g., endothelial cells, immune cells, stellate cells, pericytes, etc.) (32). This approach could permit the formation of complex, functional organoids or microtissues that include the different tumor and stromal cell populations and ECM components in functional 3D matrices and/or scaffolds (40).

Given these advantages, the organotypic system has an enormous potential to permit the more realistic analysis of tumor development, progression and sensitivity to therapy since 3D architecture, ECM composition/structure and stromal/metabolic microenvironments exert strong influences on drug efficacy. Indeed, evidence is emerging that $3 \mathrm{D}$ models with microenvironments customized to more closely mimic the tumors microenvironment are superior to both 2D and animal models $(5-7,16)$. Our findings suggest that this aspect together with its inherent capacity to easily reproduce the tumor microenvironment to produce complex co-culture conditions and perform drug schedule regimens after the tumor has developed in all its complexity, makes this type of culture the system of choice. Indeed, the further development of organotypic $3 \mathrm{D}$ culture with tissue engineering could enable the development of more complex, robust and relevant heterologous 3D tumor models to be used for designing personalized treatment. Moreover, because these complex organotypic platforms could provide drug chemosensitivity data within 9 days that is equivalent to the results generated from mouse tumor xenograft models in 50 days, the organotypic platform would be more accurate, efficient, and cost-effective and may reduce or replace animal models in the near future to identify new drug candidates, predict drug efficacy, prevent drug resistance, and improve the quality of life.

Therefore, future directions in this field will be to develop disease- or pathway-specific tissue models and adapt these models to create patient-specific systems to screen drug response in a potentially individualized manner to have direct personal relevance for each patient.

\section{Acknowledgements}

This study was supported by Associazione Italiana per la Ricerca sul Cancro (AIRC) grant no. 11348 to S.J.R. K.Z. and M.S. are fellows of Marie Curie Initial Training Network IonTraC (FP7-PEOPLE-2011-ITN grant agreement no. 289648). The SJR laboratory is part of the Italian network Istituto Nazionale Biostrutture e Biosistemi (INBB) and the project BioBoP of the Region Puglia.

\section{References}

1. Hutchinson L and Kirk R: High drug attrition rates - where are we going wrong? Nat Rev Clin Oncol 8: 189-190, 2011.

2. Cukierman E, Pankov R, Stevens DR and Yamada KM: Taking cell-matrix adhesions to the third dimension. Science 294: 1708-1712, 2001.
3. Erkan M, Hausmann S, Michalski CW, Fingerle AA, Dobritz M, Kleeff $\mathbf{J}$ and Friess H: The role of stroma in pancreatic cancer: Diagnostic and therapeutic implications. Nat Rev Gastroenterol Hepatol 9: 454-467, 2012.

4. Neesse A, Michl P, Frese KK, Feig C, Cook N, Jacobetz MA Lolkema MP, Buchholz M, Olive KP, Gress TM, et al: Stromal biology and therapy in pancreatic cancer. Gut 60: 861-868, 2011.

5. Partensky C: Toward a better understanding of pancreatic ductal adenocarcinoma: Glimmers of hope? Pancreas 42: 729-739, 2013.

6. Oettle H: Progress in the knowledge and treatment of advanced pancreatic cancer: From benchside to bedside. Cancer Treat Rev 40: 1039-1047, 2014.

7. Rahib L, Smith BD, Aizenberg R, Rosenzweig AB, Fleshman JM and Matrisian LM: Projecting cancer incidence and deaths to 2030: The unexpected burden of thyroid, liver, and pancreas cancers in the United States. Cancer Res 74: 2913-2921, 2014.

8. Coleman SJ, Watt J, Arumugam P, Solaini L, Carapuca E, Ghallab M, Grose RP and Kocher HM: Pancreatic cancer organotypics: High throughput, preclinical models for pharmacological agent evaluation. World J Gastroenterol 20: 8471-8481, 2014.

9. Hwang CI, Boj SF, Clevers H and Tuveson DA: Preclinical models of pancreatic ductal adenocarcinoma. J Pathol 238: 197-204, 2016.

10. Cardone RA, Greco MR, Zeeberg K, Zaccagnino A, Saccomano M, Bellizzi A, Bruns P, Menga M, Pilarsky C, Schwab A, et al: A novel NHE1-centered signaling cassette drives epidermal growth factor receptor-dependent pancreatic tumor metastasis and is a target for combination therapy. Neoplasia 17: 155-166, 2015.

11. Kimlin L, Kassis J and Virador V: 3D in vitro tissue models and their potential for drug screening. Expert Opin Drug Discov 8: 1455-1466, 2013.

12. Alves F, Contag S, Missbach M, Kaspareit J, Nebendahl K, Borchers U, Heidrich B, Streich R and Hiddemann W: An orthotopic model of ductal adenocarcinoma of the pancreas in severe combined immunodeficient mice representing all steps of the metastatic cascade. Pancreas 23: 227-235, 2001.

13. Chou TC: Drug combination studies and their synergy quantification using the Chou-Talalay method. Cancer Res 70: 440-446, 2010.

14. Hebner C, Weaver VM and Debnath J: Modeling morphogenesis and oncogenesis in three-dimensional breast epithelial cultures. Annu Rev Pathol 3: 313-339, 2008.

15. Yamada KM and Cukierman E: Modeling tissue morphogenesis and cancer in 3D. Cell 130: 601-610, 2007.

16. Collisson EA, Sadanandam A, Olson P, Gibb WJ, Truitt M, Gu S, Cooc J, Weinkle J, Kim GE, Jakkula L, et al: Subtypes of pancreatic ductal adenocarcinoma and their differing responses to therapy. Nat Med 17: 500-503, 2011.

17. Sipos B, Möser S, Kalthoff H, Török V, Löhr M and Klöppel G: A comprehensive characterization of pancreatic ductal carcinoma cell lines: Towards the establishment of an in vitro research platform. Virchows Arch 442: 444-452, 2003.

18. Edmondson R, Broglie JJ, Adcock AF and Yang L: Threedimensional cell culture systems and their applications in drug discovery and cell-based biosensors. Assay Drug Dev Technol 12: 207-218, 2014.

19. Navas C, Hernández-Porras I, Schuhmacher AJ, Sibilia M, Guerra $\mathrm{C}$ and Barbacid M: EGF receptor signaling is essential for k-ras oncogene-driven pancreatic ductal adenocarcinoma. Cancer Cell 22: 318-330, 2012.

20. Froeling FE, Mirza TA, Feakins RM, Seedhar A, Elia G, Hart IR and Kocher HM: Organotypic culture model of pancreatic cancer demonstrates that stromal cells modulate E-cadherin, betacatenin, and Ezrin expression in tumor cells. Am J Pathol 175: 636-648, 2009.

21. Antelmi E, Cardone RA, Greco MR, Rubino R, Di Sole F, Martino NA, Casavola V, Carcangiu M, Moro L and Reshkin SJ: $\beta 1$ integrin binding phosphorylates ezrin at T567 to activate a lipid raft signalsome driving invadopodia activity and invasion. PLoS One 8: e75113, 2013.

22. Canel M, Serrels A, Frame MC and Brunton VG: E-cadherinintegrin crosstalk in cancer invasion and metastasis. J Cell Sci 126: 393-401, 2013.

23. Clucas J and Valderrama F: ERM proteins in cancer progression. J Cell Sci 127: 267-275, 2014. 
24. Wendt MK, Taylor MA, Schiemann BJ and Schiemann WP Down-regulation of epithelial cadherin is required to initiate metastatic outgrowth of breast cancer. Mol Biol Cell 22: $2423-2435,2011$

25. Sawada K, Mitra AK, Radjabi AR, Bhaskar V, Kistner EO, Tretiakova M, Jagadeeswaran S, Montag A, Becker A, Kenny HA, et al: Loss of E-cadherin promotes ovarian cancer metastasis via alpha 5-integrin, which is a therapeutic target. Cancer Res 68 2329-2339, 2008.

26. Qin R, Smyrk TC, Reed NR, Schmidt RL, Schnelldorfer T, Chari ST, Petersen GM and Tang AH: Combining clinicopathological predictors and molecular biomarkers in the oncogenic $\mathrm{K}-\mathrm{RAS} / \mathrm{Ki} 67 / \mathrm{HIF}-1 \alpha$ pathway to predict survival in resectable pancreatic cancer. Br J Cancer 112: 514-522, 2015.

27. Kremer KN, Dudakovic A, Hess AD, Smith BD, Karp JE, Kaufmann SH, Westendorf JJ, van Wijnen AJ and Hedin KE: Histone deacetylase inhibitors target the leukemic microenvironment by enhancing a Nherf1-protein phosphatase $1 \alpha$-TAZ signaling pathway in osteoblasts. J Biol Chem 290: 29478-29492, 2015.

28. Malfettone A, Silvestris N, Paradiso A, Mattioli E, Simone G and Mangia A: Overexpression of nuclear NHERF1 in advanced colorectal cancer: Association with hypoxic microenvironment and tumor invasive phenotype. Exp Mol Pathol 92: 296-303, 2012 .

29. Troncoso M, Cuello Carrión FD, Guiñazu E, Fanelli MA, MonttGuevara M, Cabrini RL, Carón RW and Kreimann EL: Expression of NHERF1 in colonic tumors induced by 1,2-dimethylhydrazine in rats is independent of plasma ovarian steroids. Horm Cancer 2 : 214-223, 2011 .

30. Cardone RA, Bellizzi A, Busco G, Weinman EJ, Dell'Aquila ME, Casavola V, Azzariti A, Mangia A, Paradiso A and Reshkin SJ: The NHERF1 PDZ2 domain regulates PKA-RhoA-p38-mediated NHE1 activation and invasion in breast tumor cells. Mol Biol Cell 18: 1768-1780, 2007.

31. Wang B, Means CK, Yang Y, Mamonova T, Bisello A, Altschuler DL, Scott JD and Friedman PA: Ezrin-anchored protein kinase A coordinates phosphorylation-dependent disassembly of a NHERF1 ternary complex to regulate hormonesensitive phosphate transport. J Biol Chem 287: 24148-24163, 2012.
32. Cardone RA, Greco MR, Capulli M, Weinman EJ, Busco G, Bellizzi A, Casavola V, Antelmi E, Ambruosi B, Dell'Aquila ME, et al: NHERF1 acts as a molecular switch to program metastatic behavior and organotropism via its PDZ domains. Mol Biol Cell 23: 2028-2040, 2012.

33. Lee JM, Mhawech-Fauceglia P, Lee N, Parsanian LC, Lin YG, Gayther SA and Lawrenson K: A three-dimensional microenvironment alters protein expression and chemosensitivity of epithelial ovarian cancer cells in vitro. Lab Invest 93: 528-542, 2013.

34. Godugu C, Patel AR, Desai U, Andey T, Sams A and Singh M: AlgiMatrix $^{\mathrm{TM}}$ based $3 \mathrm{D}$ cell culture system as an in-vitro tumor model for anticancer studies. PLoS One 8: e53708, 2013.

35. Baker BM and Chen CS: Deconstructing the third dimension How 3D culture microenvironments alter cellular cues. J Cell Sci 125: 3015-3024, 2012.

36. Voulgari A and Pintzas A: Epithelial-mesenchymal transition in cancer metastasis: Mechanisms, markers and strategies to overcome drug resistance in the clinic. Biochim Biophys Acta 1796: 75-90, 2009.

37. Longati P, Jia X, Eimer J, Wagman A, Witt MR, Rehnmark S, Verbeke C, Toftgård R, Löhr M and Heuchel RL: 3D pancreatic carcinoma spheroids induce a matrix-rich, chemoresistant phenotype offering a better model for drug testing. BMC Cancer 13: 95, 2013.

38. Denayer S, Stöhr T and Van Roy M: Animal models in translational medicine: Validation and prediction. New Horiz Transl Med 2: 5-11, 2014.

39. Greek R and Menache A: Systematic reviews of animal models: Methodology versus epistemology. Int J Med Sci 10: 206-221, 2013.

40. Härmä V, Schukov HP, Happonen A, Ahonen I, Virtanen J, Siitari H, Åkerfelt M, Lötjönen J and Nees M: Quantification of dynamic morphological drug responses in 3D organotypic cell cultures by automated image analysis. PLoS One 9: e96426, 2014. 